\title{
Optimisation du procédé traditionnel de maltage du sorgho pour la production de boissons fermentées
}

\author{
A. P. P. KAYODÉ ${ }^{1 *}$, I. S. AHOUANSE ${ }^{1}$, S.O. KOTCHONI ${ }^{2}$ et J. D. HOUNHOUIGAN ${ }^{1}$ \\ ${ }^{1}$ Faculté des Sciences Agronomiques, Université d'Abomey-Calavi, 01 BP 526 Cotonou, Bénin. \\ ${ }^{2}$ Department of Biology, Rutgers University, Camden, New Jersey 08102, USA. \\ *Auteur correspondant, E-mail: polykap@yahoo.fr; Tel +22997870734.
}

\section{RESUME}

La présente étude a évalué les conditions d'optimisation des procédés traditionnels de maltage du sorgho au Bénin. L'objectif est d'améliorer, à l'échelle semi-industrielle, la production et la qualité du malt de sorgho. L'étude s'est déroulée en deux phases. La première phase a consisté en une enquête technologique au cours de laquelle la technologie traditionnelle de production de malt a été suivie et des échantillons de malt collectés. Dans la deuxième phase, la technologie traditionnelle a été reproduite en conditions contrôlées au laboratoire en faisant varier les différents paramètres de maltage que sont la durée de trempage et la durée de germination. Les résultats ont montré qu'il existe une grande variabilité au niveau des caractéristiques physicochimiques des malts collectés auprès des productrices de boissons maltées. L'analyse de variance a révélé que la durée de trempage et la durée de germination ont un effet significatif sur l'extrait sec, le $\mathrm{pH}$, le pouvoir diastasique et le rapport $\alpha / \beta$-amylasique des malts dérivés. Une analyse en composantes principales a été effectuée sur les caractéristiques des malts produits en conditions contrôlées et les conditions optimales de production de malt de qualité adéquate ont été établies.

(C) 2011 International Formulae Group. All rights reserved.

Mots clés : Sorgho, malt, pouvoir diastasique, germination, fermentation, optimisation.

\section{INTRODUCTION}

Le sorgho est une plante céréalière appartenant à la famille des graminées et à la tribu des andropogonés. L'espèce Sorghum Bicolor est la plus cultivée dans les zones intertropicales de l'Afrique (Dicko et al., 2005). Le sorgho peut être utilisé directement ou sous forme maltée et/ou fermentée dans la préparation de plusieurs aliments traditionnels tels que les bouillies, les pâtes et les boissons alcoolisées et non alcoolisées (Dicko et al., 2006). Trois principaux groupes d'aliments à base de sorgho ont été recensés au Bénin; il s'agit : des boissons (tchoukoutou; chakpalo), des pâtes (ogui, akassa, dibou) et des bouillies (gowé, koko, sorou) (Nago et Hounhouigan, 1998 ; Kayodé et al., 2005). Le malt de sorgho est utilisé pour la production de différents aliments tels que le tchoukoutou, le chakpalo et le gowé. Plus récemment, Kayodé (2006) a suggéré l'utilisation du malt de sorgho pour la production des bouillies infantiles compte tenu de la valeur nutritionnelle élevée des produits dérivés. Le maltage consiste en trois 
étapes à savoir: le trempage, la germination et le séchage. Cette technique permet d'enrichir les céréales en enzymes hydrolytiques telles que la bêta-amylase et l'alpha-amylase, en sucres, en acides aminés libres et en vitamines améliorant ainsi la qualité technologique et nutritionnelle des produits dérivés (Sripriya et al., 1997 ; Elmaki et al., 1999 ; MbithiMwikya et al., 2000 ; Mbofung et Fombang, 2003). Le maltage présente aussi l'avantage de réduire la teneur en facteurs antinutritionnels des grains de céréales et d'induire une augmentation de la biodisponibilité des minéraux comme le fer et le zinc (Traoré et al., 2004 ; Kayodé, 2006). De plus, l'addition de malt aux farines infantiles permet d'avoir des bouillies de densité énergétique élevée (Trèche, 1995 ; Elkhalil, 2001 ; Tou et al., 2003 ; Towo et al., 2003). Au Bénin, on note une baisse de la productivité et de la qualité du malt au niveau des entreprises artisanales de production de malt et produits dérivés à cause d'un certain nombre de contraintes technologiques: pénibilité et longue durée des procédés de fabrication et perte de savoir-faire. Dans la majorité des cas, il a été observé l'addition de sucre à la farine de sorgho pour lui conférer le goût sucré normalement obtenu par maltage (Michodjehoun-Mestres et al., 2005). De telles pratiques compromettraient la qualité nutritionnelle du produit final. Des études ont été réalisées sur la qualité des produits dérivés du malt de sorgho au Bénin (Kayodé, 2006). Mais aucune étude ne s'est penchée sur la qualité du malt destiné à la production des aliments maltés. La présente étude vise à améliorer la qualité des malts de sorgho pour la production de produits maltés. De façon spécifique, cette étude se propose de (i) capitaliser les connaissances locales sur les techniques de production du malt de sorgho; (ii) évaluer la qualité technologique du malt de sorgho et; (iii) définir les conditions opératoires optimales permettant la production de malt de qualité.

\section{MATERIEL ET METHODES Matériel végétal}

Les grains de sorgho utilisés dans cette étude ont été achetés au marché Dantokpa à Cotonou au Bénin. Il s'agit du sorgho rouge (Sorghum bicolor) généralement apprécié et utilisé par les productrices de boissons à base de malt de sorgho.

\section{Dispositif expérimental}

Le dispositif expérimental utilisé comporte deux facteurs $(\mathrm{k}=2)$ à savoir le trempage et la germination. Ce dispositif a permis d'estimer l'effet du trempage et de la germination sur le pouvoir diastasique, le rapport $\alpha / \beta$ amylasique, l'extrait sec et le $\mathrm{pH}$ du malt du sorgho. Dans ce dispositif, le niveau de trempage a varié de $8 \mathrm{~h}$ à $24 \mathrm{~h}$ et celui de la germination de $24 \mathrm{~h}$ à $72 \mathrm{~h}$. Le dispositif a généré 12 observations (Tableau 1).

\section{Production expérimentale du malt}

Environ $500 \mathrm{~g}$ de grains de sorgho propres ont été trempés dans de l'eau de robinet à la température ambiante $\left(28-30{ }^{\circ} \mathrm{C}\right)$ pendant $8 \mathrm{~h}, 10 \mathrm{~h}, 18 \mathrm{~h}$ et $24 \mathrm{~h}$. Après trempage, les grains ont été égouttés et étalés sur un sac de jute préalablement disposé dans un panier en osier tressé, et laissés en germination à la température ambiante (28-30 ${ }^{\circ} \mathrm{C}$ ). Les grains en germination ont subi deux arrosages quotidiens (matin et soir) par aspersion d'eau. Le séchage des échantillons a été fait à l'aide du séchoir de marque HERAEUS. Il s'agit d'un séchoir à air chaud circulant. Les claies utilisées lors du séchage sont des plateaux en acier inoxydable. Les malts ont été séchés à $45^{\circ} \mathrm{C}$ afin de limiter les pertes en enzymes amylolytiques.

\section{Suivi des opérations et prélèvement des échantillons}

En vue de caractériser le malt traditionnel, des échantillons de malt traditionnel ont été collectés sur 10 sites de 
productions de gowé à Cotonou en République du Bénin. Ces échantillons ont été caractérisés au laboratoire pour leur teneur en eau, le pouvoir diastasique, le rapport $\alpha / \beta$ amylasique, l'extrait sec et le $\mathrm{pH}$. Lors de la production expérimentale de malt, des échantillons ont été prélevés à différentes heures conformément au dispositif expérimental (Tableau 2).

\section{Analyses physico-chimiques \\ Teneur en eau}

La teneur en eau des échantillons a été déterminée par la méthode AACC (1984).

\section{Pouvoir diastasique}

Le pouvoir diastasique a été déterminé selon la méthode proposée par Giamarchi (1992). Elle consiste à faire agir un extrait enzymatique sur une solution d'amidon. Les sucres réducteurs et les fonctions réductrices ainsi libérés sont dosés par colorimétrie (540 $\mathrm{nm})$ en présence de l'acide 3-5 dinitrosalicylique. Une gamme d'étalon maltose est réalisée et dosée dans les mêmes conditions que précédemment. L'absorbance est mesurée à $540 \mathrm{~nm}$. L'activité enzymatique est déterminée en se référant à la courbe d'étalonnage et est exprimée en milliéquivalent maltose $/ \mathrm{min} / \mathrm{g}$ de malt.

\section{Activités $\alpha$ et $\boldsymbol{B}$-amylasique des malts traditionnels}

L'activité $\quad \alpha$-amylasique a été déterminée selon la méthode proposée par Giamarchi (1992), selon le même principe que celui du pouvoir diastasique, après extraction des enzymes avec une solution de $\mathrm{CaCl}_{2}(3,3 \mathrm{~g} / \mathrm{l})$ et inactivation de la $\beta$-amylase à $68{ }^{\circ} \mathrm{C}$ pendant $15 \mathrm{~min}$. L'activité $\beta$ amylasique est déduite par la différence entre le pouvoir diastasique au $\mathrm{CaCl}_{2}$ et l'activité amylasique.

\section{Détermination de l'extrait sec (ES) des malts}

L'extrait sec est la teneur en matières sèches solubles contenue dans le malt. Il a été obtenu selon un programme de brassage au Rapid Visco Analyser (RVA) qui comprend 4 phases principales à savoir: l'extraction d'enzyme, la gélatinisation de l'amidon du culot, la saccharification et la filtration. Une prise d'essai de $2 \mathrm{ml}$ de filtrat évaporée dans une capsule est séchée pendant $24 \mathrm{H}$ à 100 ${ }^{\circ} \mathrm{C}$. La teneur en (ES) est exprimée par rapport à $100 \mathrm{~g}$ de malt sec (Kayodé et al., 2001).

\section{Détermination du pH}

La méthode utilisée est celle de Nout et al. (1989) modifiée. Environ $10 \mathrm{~g}$ de farine de malt obtenue par mouture au moulin Retsch (Retsch GmbH, Haan, Germany) équipé d'un tamis de maille $1 \mathrm{~mm}$, sont prélevés dans un bécher et additionnés de $20 \mathrm{ml}$ d'eau distillée. Le mélange est homogénéisé à l'aide d'un agitateur magnétique et le $\mathrm{pH}$ est mesuré. Le pH-mètre utilisé est étalonné avec les solutions tampons de $\mathrm{pH}=4$ et $\mathrm{pH}=7$. La valeur du $\mathrm{pH}$ est lue directement sur le cadran de l'appareil.

\section{Analyses statistiques}

Les données ont été analysées en utilisant le programme statistique SPSS 12.0 (SPSS, Chicago, IL, USA). L'analyse de variance univariée a été utilisée pour évaluer la différence entre les moyennes. Le niveau de signification retenu est de $5 \%(\mathrm{p}<0,05)$.

\section{RESULTATS}

Qualité technologique du malt traditionnel

La qualité technologique des malts traditionnels a été évaluée en appréciant leur pouvoir diastasique, leur extrait sec, le $\mathrm{pH}$ et le rapport $\alpha / \beta$ amylasique (Tableau 1). Les pouvoirs diastasiques des échantillons collectés varient de 55,5 à 152,8 UPD avec un coefficient de variation de $37 \%$. Au niveau de l'extrait sec soluble par contre, les valeurs varient de 52,7 à 64,4 g/100g de malt avec un faible coefficient de variation $(\mathrm{CV}=8 \%)$. Pour les différents malts collectés, le rapport $\alpha / \beta$ est généralement faible et est de l'ordre de 0,42 à 2,95. Cela signifie que pour la plupart 
de ces malts, la teneur en $\beta$ amylase est supérieure à celle de l' $\alpha$ amylase, exception faite cependant des malts $\mathrm{P} 4$ et $\mathrm{P} 7$. Tous les malts traditionnels ont un $\mathrm{pH}$ se rapprochant de 6 confirmant ainsi les résultats obtenus. Sachant que la $B$-amylase a un $\mathrm{pH}$ de l'ordre de 6 , alors nous pourrions nous attendre à de fortes activités de la $B$-amylase dans les malts produits.

\section{Qualité technologique des malts expérimentaux}

Différentes durées de trempage et de germination ont été combinées et leurs effets sur le pouvoir diastasique, l'extrait sec, le $\mathrm{pH}$ et le rapport $\alpha / \beta$-amylase ont été mesurés. Les résultats obtenus sont présentés dans le Tableau 2.

\section{Effet du maltage sur le pouvoir diastasique des malts}

La Figure 1a présente l'évolution du pouvoir diastasique en fonction de la durée de trempage et de la durée de germination. L'activité des amylases hydrosolubles s'accroît de façon significative avec l'augmentation de la durée de trempage et de la germination. En effet, le PD augmente graduellement de 39,3 à 181,8 quand la durée de germination passe de 24 à $72 \mathrm{~h}$. Pour ce qui est de l'effet de la durée de trempage, le PD s'accroît dans un premier temps de 93,6 à 172,3 quand le trempage évolue de 8 à $18 \mathrm{~h}$ (Figure 1a). Le PD tend à diminuer en passant de 172,3 à 129,6 UPD lorsque la durée de trempage croît de $18 \mathrm{~h}$ à $24 \mathrm{~h}$. Il a été observé que le malt ayant le pouvoir diastasique (PD) le plus élevé a un PD de 152,8 UPD

\section{Effet du maltage sur le Rapport $\alpha / \beta$ - amylase}

L'importance de ce paramètre réside dans la connaissance de la contribution relative de chacune des amylases principales à la dégradation de la structure de l'amidon. L'analyse de la Figure $1 \mathrm{~b}$ révèle qu'en moyenne le rapport $\alpha / \beta$-amylase diminue de façon significative avec l'augmentation de la durée de trempage. Par contre, la durée de germination n'influence pas significativement ce ratio. Le test de Newman-Keuls révèle que tous les malts sont semblables, à l'exception du malt obtenu à $8 \mathrm{~h}$ de trempage et $72 \mathrm{~h}$ de germination qui présente un rapport $\alpha / \beta$ amylase très élevé $(6,4)$.

\section{Effet du maltage sur l'extrait sec}

L'extrait sec est la quantité de matières sèches solubles du malt. La Figure 1c montre la variation de l'extrait sec en fonction des différentes durées de trempage et de germination. L'extrait sec des malts augmente significativement en fonction de la durée de germination et de trempage. L'extrait sec de 8 $\mathrm{h}$ et celui de $10 \mathrm{~h}$ de trempage sont similaires et sont respectivement de 57,7 et $59,3 \mathrm{~g} / 100 \mathrm{~g}$ de malts. Ces deux malts présentent de faibles valeurs par rapport à ceux de $18 \mathrm{~h}$ et de $24 \mathrm{~h}$ de trempage qui sont aussi par ailleurs très proches.

\section{Optimisation des paramètres de maltage}

Dans le souci de déduire de l'ensemble de nos résultats expérimentaux les durées optimales de germination et de trempage, nous avons réalisé une analyse en composantes principales (ACP). Cette ACP a pris en compte les valeurs de toutes les combinaisons de durées de trempage et de durées de germination pour les variables étudiées (Figure 2). Dans cette ACP, l'axe 1 est expliqué à $23 \%$ par le pouvoir diastasique, $21 \%$ par le pH et $20 \%$ par l'extrait sec. L'axe 2 est expliqué à $46,3 \%$ par le rapport $\alpha / \beta$ amylase et à $33 \%$ par la teneur en matière sèche (Figure 3). Ces niveaux d'explications étant supérieurs à $20 \%$, cette ACP peut être considérée comme valide. 
Tableau 1: Caractéristiques technologiques des malts traditionnels.

\begin{tabular}{ccccc}
\hline Producteur & $\begin{array}{c}\text { Pouvoir } \\
\text { diastasique }(\mathbf{U P D})^{\mathbf{a}}\end{array}$ & $\begin{array}{c}\text { Extrait sec } \\
(\mathbf{g} / \mathbf{1 0 0 g})\end{array}$ & $\mathbf{p H}$ & $\begin{array}{c}\text { Rapport } \boldsymbol{\alpha} / \boldsymbol{\beta}- \\
\text { amylase }\end{array}$ \\
\hline P1 & $81,29 \pm 6,0$ & $56,35 \pm 0,3$ & $5,92 \pm 0,01$ & 0,72 \\
P2 & - & $64,44 \pm 0,6$ & $5,86 \pm 0,01$ & 0,55 \\
P3 & $152,81 \pm 3,5$ & $60,27 \pm 2,7$ & $5,85 \pm 0,01$ & 0,8 \\
P4 & $55,32 \pm 5,2$ & $52,65 \pm 2,0$ & $5,97 \pm 0,01$ & 1,31 \\
P5 & $54,49 \pm 3,5$ & $54,15 \pm 2,2$ & $6,04 \pm 0,01$ & 0,33 \\
P6 & $67,42 \pm 2,8$ & $54,57 \pm 0,9$ & $5,93 \pm 0,01$ & 0,21 \\
P7 & $104,89 \pm 4,4$ & $63,68 \pm 2,2$ & $5,89 \pm 0,02$ & 2,95 \\
P8 & $98,22 \pm 1,8$ & $61,83 \pm 2,8$ & $5,46 \pm 0,02$ & 0,72 \\
P9 & $139,67 \pm 6,3$ & $63,04 \pm 0,9$ & $5,87 \pm 0,01$ & 0,42 \\
P10 & $125,89 \pm 9,0$ & $55,37 \pm 0,7$ & $5,87 \pm 0,0$ & 0,7 \\
\hline Moyenne & 97,78 & 58,64 & 5,87 & 0,87 \\
\hline CV (\%) & 37 & 8 & 3 & 91 \\
\hline${ }^{2}$ Unité Pouvoir Diastasique (U PD) & & &
\end{tabular}

Tableau 2: Caractéristiques technologiques des malts produits en conditions expérimentales.

\begin{tabular}{lcccccc}
\hline \multirow{2}{*}{ Traitements } & & $\begin{array}{c}\text { Matière } \\
\text { sèche }(\boldsymbol{\%})\end{array}$ & $\begin{array}{c}\text { Extrait } \\
\text { sec } \\
(\mathbf{g} / \mathbf{1 0 0 g})\end{array}$ & $\begin{array}{c}\text { Pouvoir } \\
\text { diastasique } \\
(\mathbf{U P D})\end{array}$ & $\begin{array}{c}\text { Rapport } \\
\boldsymbol{\alpha} / \boldsymbol{\beta} \text {-amylase }\end{array}$ & pH \\
\hline Trempage (h) & Germination(h) & & & & & \\
\hline 8 & 24 & 93,07 & 41,62 & 38,18 & 2,56 & 6,16 \\
8 & 48 & 93,12 & 64,94 & 100,71 & 2,78 & 5,9 \\
8 & 72 & 92,22 & 66,6 & 141,83 & 6,36 & 5,93 \\
10 & 24 & 93,52 & 43,77 & 18,39 & 2,04 & 6,17 \\
10 & 48 & 94,66 & 65,42 & 99,08 & 1,585 & 5,88 \\
10 & 72 & 94,88 & 68,65 & 166,86 & 0,7 & 5,73 \\
18 & 24 & 90,82 & 53,16 & 88,68 & 1,49 & 5,81 \\
18 & 48 & 93,3 & 69,2 & 198,25 & 3,735 & 5,77 \\
18 & 72 & 91,49 & 70,37 & 229,83 & 1,615 & 5,44 \\
24 & 24 & 94,39 & 50,8 & 11,9 & 0,16 & 6,02 \\
24 & 48 & 94,15 & 68,65 & 188,32 & 0,96 & 5,75 \\
24 & 72 & 93,72 & 68,51 & 188,62 & 1,29 & 5,67 \\
& & & & & & \\
\hline
\end{tabular}

Tableau 3: Analyse de variance montrant l'effet des facteurs de transformations sur les caractéristiques de qualité du malt.

\begin{tabular}{lccccc}
\hline \multirow{2}{*}{ Facteurs } & $\begin{array}{c}\text { Matière } \\
\text { sèche }\end{array}$ & Extrait sec & $\begin{array}{c}\text { Pouvoir } \\
\text { diastasique }\end{array}$ & $\begin{array}{c}\text { Rapport } \boldsymbol{\alpha} / \boldsymbol{\beta}- \\
\text { amylase }\end{array}$ & pH \\
\hline Trempage & 3,42 & $30,91^{*}$ & $818,42^{*}$ & $9,42^{*}$ & $13,91^{*}$ \\
Germination & 0,72 & $641,35^{*}$ & $4366,0^{*}$ & 1,64 & $28,01^{*}$ \\
Interaction & 0,48 & $6,06^{*}$ & $138,0^{*}$ & $3,51^{*}$ & 1,78 \\
\hline$*$ Effet significatif au seuil de $5 \%$ & & &
\end{tabular}



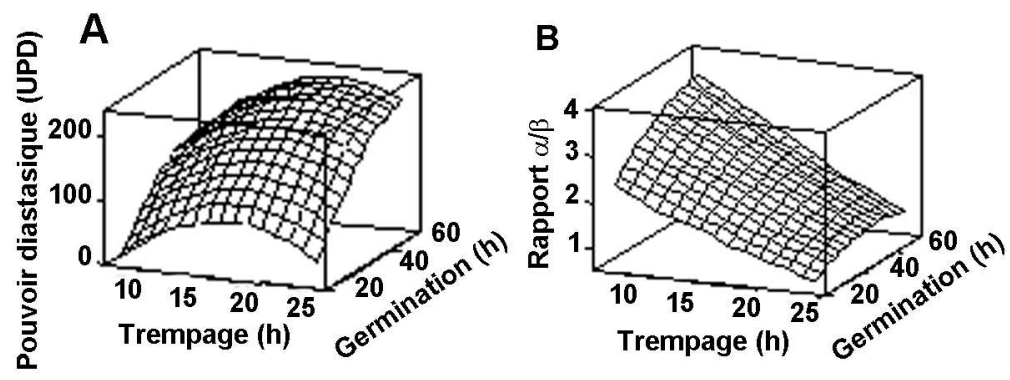

C

D
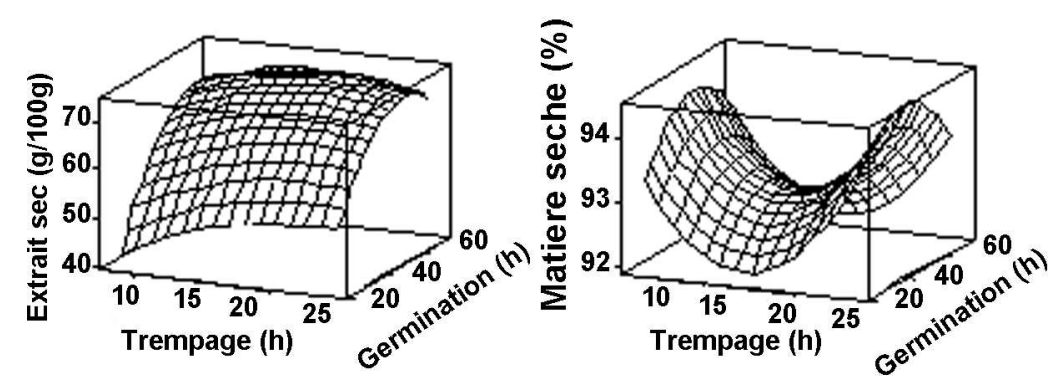

Figure 1 : Courbes de surface de réponse montrant les effets du trempage et de la germination sur (a) le pouvoir diastasique, (b) le rapport $\alpha / \beta$-amylasique, (c) l'extrait sec et (d) le pH du malt de sorgho.

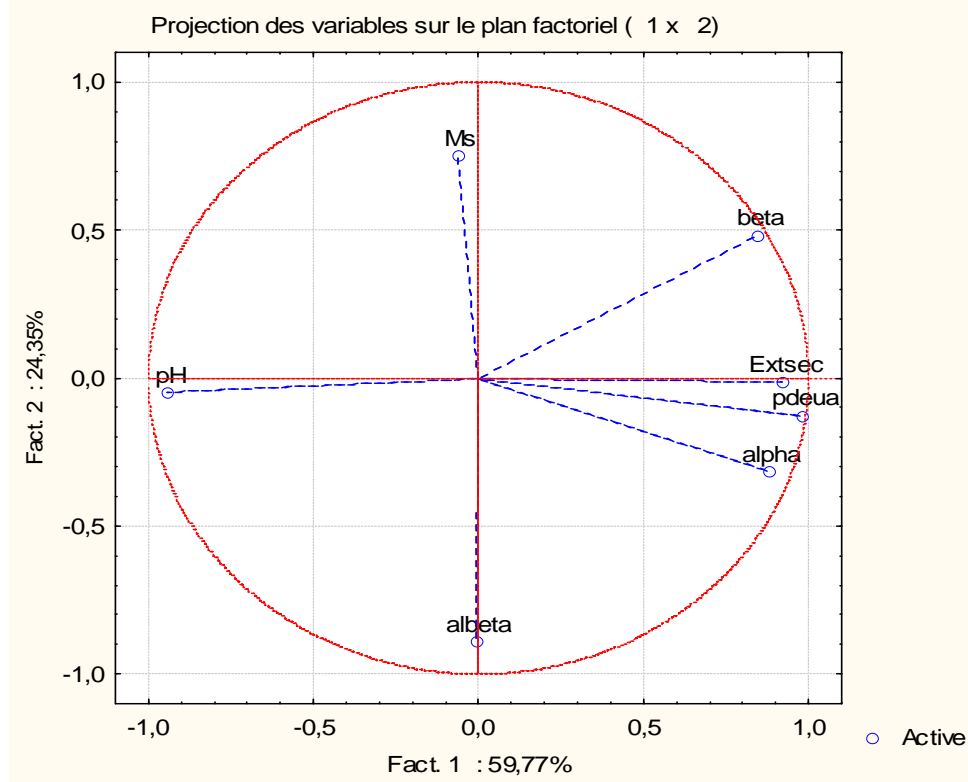

Figure 2: Projection des variables sur plan bidimentionnel. Pdeua $=$ pouvoir diastasique Albeta $=$ rapport alpha/bêta. 


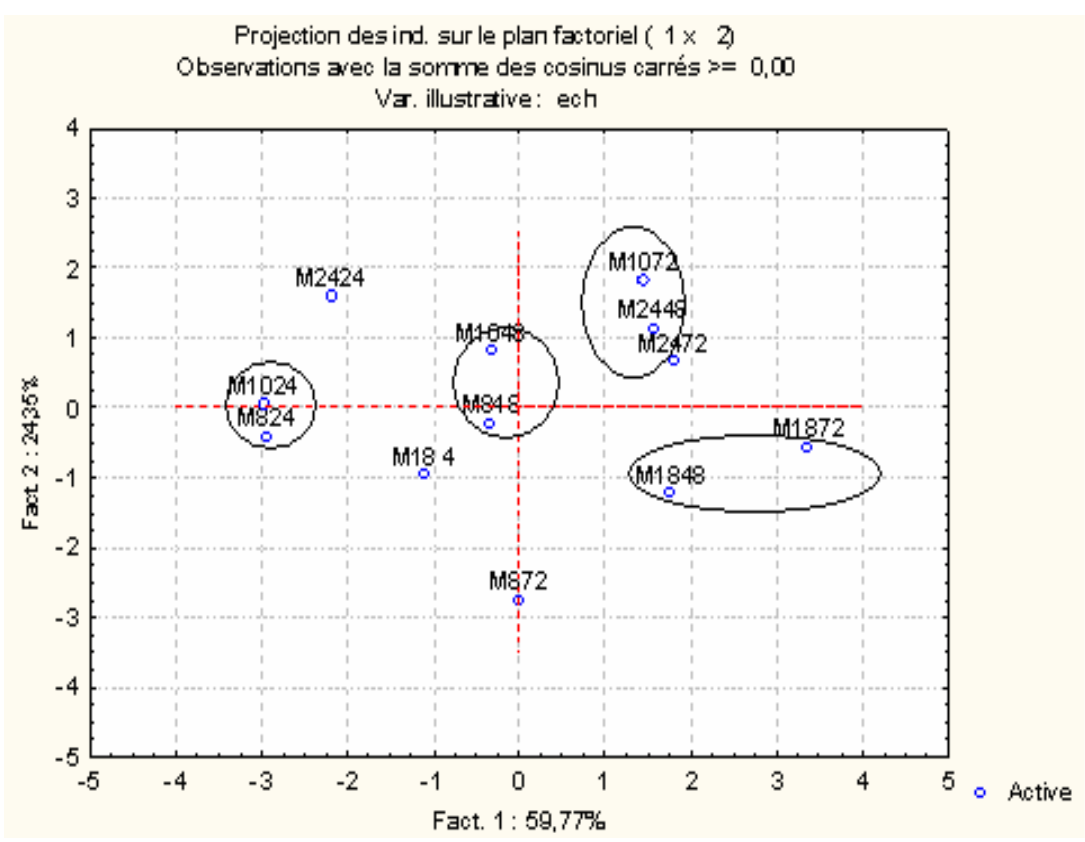

Figure 3: Projection sur plan ACP des malts produits en conditions contrôlées.

\section{DISCUSSION}

Le pouvoir diastasique (PD) du malt désigne l'activité totale de toutes les amylases du produit dans du solvant aqueux. Il indique l'activité totale des amylases hydrosolubles. Il existe une grande variabilité au niveau du pouvoir diastasique des échantillons de malt traditionnels collectés à Cotonou. Ce constat rejoint celui de Traoré et al. (2004) et Tchuenbou (2006) qui ont observé une grande variation au niveau des activités amylasiques des malts traditionnels produits au Burkina Faso. Ceci peut s'expliquer par la variation de la durée des opérations d'une productrice à l'autre et aussi par la non-maitrise des facteurs influençant le maltage par les productrices. En effet, d'une productrice à une autre et au niveau d'une même productrice, les pratiques varient d'une production à une autre. La plupart des malts présentent des extraits secs solubles relativement faibles par rapport à ceux obtenus par Demuyakor et Ohta (1992) et Tchuenbou (2006). Seul le malt P2 dont l'extrait sec est le plus élevé $(64,4 \mathrm{~g} / 100 \mathrm{~g}$ de malt), est proche des malts obtenus par Tchuenbou (2006) sur du sorgho collecté au Bénin et au Burkina Faso (64 à 80 g/100 g).
Par contre, ce malt présente un extrait sec plus faible que ceux des malts produits par Demuyakor et Yoshiyuki (1992) (66 à 77 $\mathrm{g} / 100 \mathrm{~g}$ de malt). La plupart des malts traditionnels présente une teneur en $\beta$-amylase supérieure à celle de l' $\alpha$-amylase, exception faite cependant des malts P4 et P7. Ces résultats s'opposent à ceux rapportés par Aisien (1982), qui a indiqué que l' $\alpha$-amylase est l'enzyme la plus présente et la plus active dans le malt de sorgho. Ces résultats pourraient être expliquées par les mêmes raisons évoquées au niveau du pouvoir diastasique, notamment, la non-maîtrise des différents facteurs influençant le maltage. De plus, la forte activité $\beta$-amylasique constatée au niveau des malts s'expliquerait par les valeurs de $\mathrm{pH}$ des produits. En effet, il a été remarqué que le malt traditionnel a un $\mathrm{pH}$ variant de 5,5 à $6,0(\mathrm{CV}=3 \%)$. Or, le $\mathrm{pH}$ optimum d'activation de la $B$-amylase varie entre 5 et 6 alors que celui de l' $\alpha$-amylase est de 5. En vue d'optimiser la qualité du malt, une étude expérimentale a été conduite au laboratoire en combinant différents temps de trempage et de germination. Il est apparu que la durée optimale de trempage permettant une 
bonne activité amylasique pourrait être de 18 h. Quant à la germination, le maximum d'activité amylasique est obtenu à $72 \mathrm{~h}$ dans le malt de sorgho. Sur cette base, les durées de trempage de $18 \mathrm{~h}$ et de germination de $72 \mathrm{~h}$ semblent être l'optimum qui permet de développer une bonne activité amylasique en conditions d'expérimentation. Il a été précédemment rapporté que la durée de germination optimale de sorgho pour la production de malt fluctue entre 3 et 7 jours (Novellie, 1986; Agu et Palmer, 1999). Le rapport $\alpha / \beta$-amylase renseigne sur la contribution relative de chacune des amylases principales à la dégradation de la structure de l'amidon. Au cours du trempage, il y a une diminution de l'activité $\alpha$-amylasique au profit de l'activité $\beta$-amylase. Ces résultats sont conformes à ceux de Tchuenbou (2006), qui a aussi observé une diminution du rapport $\alpha / \beta$ au cours du trempage. Par ailleurs, le malt obtenu à $8 \mathrm{~h}$ de trempage et $72 \mathrm{~h}$ de germination présente un rapport $\alpha / \beta$-amylase très élevé $(6,4)$. Ce type de malt possède les caractéristiques d'un malt qui pourrait être utilisé dans l'alimentation infantile en ce sens qu'il pourra permettre une réduction accrue de la viscosité des bouillies et augmenter leur densité énergétique. En effet, Traoré et al. (2004) et Tchuenbou (2006) ont rapporté que des fortes activités $\alpha$-amylasiques favorisent la réduction de la viscosité des bouillies dérivées. L'extrait sec est un paramètre très important pour la production de boissons maltées comme le gowé puisqu'il renseigne sur les matières solubles du produit. De même, le goût est un critère important de consommation des boissons maltées comme le gowé et il est positivement corrélé à l'extrait sec du produit (Dalodé, 2008). L'extrait sec de $8 \mathrm{~h}$ et celui de $10 \mathrm{~h}$ de trempage sont similaires et sont respectivement de 57,7 et $59,3 \mathrm{~g} / 100 \mathrm{~g}$ de malts. Ces deux malts présentent de faibles valeurs par rapport à ceux de $18 \mathrm{~h}$ et de $24 \mathrm{~h}$ de trempage qui sont aussi par ailleurs très proches. Les résultats d'optimisation synthétisés dans la Figure 3 montrent que le malt produit après $18 \mathrm{~h}$ de trempage et $72 \mathrm{~h}$ de germination possède les meilleures caractéristiques. En particulier, ce malt possède un bon pouvoir diastasique $(229,83)$, un $\mathrm{pH}$ adéquat $(5,44)$, une bonne quantité d'extrait sec $(70,37)$. Au surplus, il a une activité $\alpha$-amylasique et une activité $\beta$ amylasique raisonnables. L'analyse de corrélation de Pearson a révélé que le pouvoir diastasique est positivement corrélé avec l'extrait sec de manière significative $(\mathrm{P}<$ 0,05). De même l'extrait sec est fortement et positivement corrélé avec les activités $\alpha$ amylasique et $\beta$-amylasique. Le $\mathrm{pH}$ est négativement corrélé avec l'extrait sec et le pouvoir diastasique. Par ailleurs, on note que l'activité $\quad \alpha$-amylasique, l'activité $\quad \beta$ amylasique et le $\mathrm{pH}$ sont corrélés négativement avec la durée de trempage. Enfin, l'extrait sec, l'activité $\alpha$-amylasique, l'activité $\beta$-amylasique et le pouvoir diastasique sont corrélés positivement avec la durée de germination.

\section{Conclusion}

La présente étude nous a permis d'évaluer l'effet des différents paramètres de maltage (durées de trempage et de germination) sur la qualité du malt de sorgho. On a pu observer une grande variabilité au niveau du pouvoir diastasique des malts traditionnels. Il est aussi apparu que les durées de trempage et de germination ont un effet significatif sur le pouvoir diastasique, l'extrait sec, le rapport $\alpha / \beta$-amylase et le $\mathrm{pH}$ des malts. Le malt obtenu en combinant $18 \mathrm{~h}$ de trempage et $72 \mathrm{~h}$ de germination présente une activité $\alpha$-amylasique élevée et une activité $\beta$ amylasique moyenne avec un rapport $\alpha / \beta$ amylase de 1,6. Ce malt possède le pouvoir diastasique le plus élevé (229,8 UPD). Les caractéristiques de ce malt semblent convenables pour la production de produits maltés.

\section{REMERCIEMENTS}

Les auteurs remercient le Forum global pour la recherche agricole (GFAR), l'association Agropolis et le Ministère français des Affaires Etrangères (MAE) pour leur appui financier à travers le projet DURAS. 


\section{REFERENCES}

AACC. 1984. Approved Methods of the American Association of Cereals Chemists (8th edn). St Paul MN: USA.

Agu RC, Palmer GH. 1999. Comparative development of soluble nitrogen in the malts of barley and sorghum. Process Biochem., 35: 497-502.

Aisien AO. 1982. Enzymatic modification of Sorghum endosperm during seedling growth and malting. J. Sci. Food Agric., 33: 754-759.

Demuyakor B, Ohta Y. 1992. Malt characteristics of Sorghum vulgare varieties from Ghana. J. Sci. Food Agric., 59: 457-462.

Dicko MH, Gruppen H, Traoré AS, Voragen AGJ, Berkel WJH. 2005. Sorghum grain as human food in africa: relevance of content of starch and amylase activities. Afr. J. Biotechnol., 5: 384-395.

Elkhalil EAI, El Tinay AH, Mohamed BE, Elsheikh EAE. 2001. Effect of malt pretreatment on phytic acid and in vitro protein digestibility of sorghum flour. Food Chem., 72: 29-32.

Elmaki HB, Babiker EE, El Tinay AH. 1999. Changes in chemical composition, grain malting, starch and tannin contents and protein digestibility during germination of sorghum cultivars. Food Chem., 64: 331336.

Giamarchi P. 1992. Dosage de l'activité alpha et béta-amylasique. In Methodes d'Analyse, Herzele P (ed). Laboratoire de Nutrition : Brazaville-Congo ; 1-8.

Kayodé APP, Hounhouigan DJ, Akissoe N, Meot JM, Mestres C. 2004. Influence de différentes conditions de pré-cuisson sur la qualité des farines et pâtes de cossettes d'igname (Dioscorea cayenensisrotundata). Ann. Sci. Agron. Benin, 6: 151-161.

Kayodé APP, Adegbidi A, Linnemann AR, Hounhouigan JD. 2005. Quality of farmers' varieties of sorghum and derived foods as perceived by consumers in Benin. Ecol. Food Nutr., 44: 271-294.
Kayodé APP. 2006. Diversity, users' perception and food processing of sorghum: implications for dietary iron and zinc supply. $\mathrm{PhD}$ thesis, Wageningen university, Wageningen, The Netherlands, p.152.

Mbithi-Mwikya S, van Camp J, Yiru Y, Huyghebaert T. 2000. Nutrient and Antinutrient changes in finger millet (Eleusine Coracan) during sprouting. LWT-Food Sci.Technol., 33: 9-14.

Mbofung CM, Fombang EN. 2003. Improving the digestibility and avalaibility of nutrients from sorghum flour improved malting techniques. In $2^{\text {nd }}$ International workshop: food-based approaches for healthy nutrition. Ouagadougou, 489-501.

Michodjehoun-Mestres L, Hounhouigan DJ, Dossou J, Mestres C. 2005. Physical, chemical and microbiological changes during natural fermentation of gowé, a sprouted or non sprouted sorghum beverage from West Africa. Afr. J. Biotechnol., 4: 487-496.

Nago M, Hounhouigan J. 1998. La Transformation Alimentaire Traditionnelle des Céréales au Bénin. Les Publications du CERNA; 152.

Nout MJR, Rombouts FM, Havelaar A. 1989. Effect of accelerated natural lactic fermentation of infant food ingredients on some pathogenic microorganisms. Int. J. Food Microbiol., 8: 351-361.

Novellie L, De Schaepdrijver P. 1986. Modern developments in traditional African beers. Progr. Industr. Microbiol., 23: 74-157.

Sripriya G, Antony U, Candra TS. 1997. Changes in carbohydrate, free amino acids, organic acids, phytate and $\mathrm{HCl}$ extractability of minerals during germination and fermentation of finger millet (Eleusine corocana). Food Chem., 58: 345-350.

Tchuenbou F. 2006. Maltage du mil et du sorgho: Mise au point d'outils et de méthodes. Aptitude au maltage de quelques variétés de sorgho du Burkina et 
du Bénin. MSc. thesis, Montpellier, France, p.130.

Tou EH, Mouquet C, Guyot JP, Traore AS, Trèche S. 2003. Essai de modification du procédé traditionnel de fabrication des bouillies de mil fermenté bensaalga en vue d'augmenter leur densité énergétique. In $2^{\text {nd }}$ International workshop: food-based approaches for healthy nutrition. Ouagadougou : 517-524.

Towo E, Svanberg U, Ndossi GD. 2003. Effect of grain pre-treatement on different extractable phenolic groups in cereals and legumes commonly consumed in Tanzania. J. Sci. Food Agric., 83: 980986.

Traore T, Mouquet C, Icard-Verniere C, Traore AS, Treche S. 2004. Changes in nutrient composition, phytate and cyanide contents and alpha-amylase activity during cereal malting in small production units in Ouagadougou (Burkina Faso). Food Chem., 88: 105-119.

Trèche S. 1995. Techniques pour augmenter la densité énergétique des bouillies. In L'Alimentation de Complément du Jeune Enfant, Trèche S, de-Benoist B, Benbouzid D, Verster A, Delpeuch F (eds). ORSTOM éditions: Paris ; 123146.

Vieira-Dalodé GA. 2008. Etude de la fermentation de la farine de sorgho pour la production du gowé au Bénin: aspects physico-chimiques et microbiologiques. PhD thesis, Université d'Abomey-Calavi, Abomey-Calavi, p.70. 